\title{
Derechos, calidad de vida y división social del espacio en el Distrito Metropolitano de Quito
}

Augusto Barrera. Pontificia Universidad Católica del Ecuador (PUCE), Quito, Ecuador.

Pablo Cabrera-Barona. Facultad Latinoamericana de Ciencias Sociales (FLACSO), Quito, Ecuador.

Pablo Velasco-Oña. Facultad Latinoamericana de Ciencias Sociales (flacso), Quito, Ecuador.

RESUMEN | Este trabajo explora la calidad de vida en el Distrito Metropolitano de Quito (DMQ) mediante la construcción de un índice multidimensional, con el que se analizan sus 65 parroquias. El índice sintetiza determinantes de la división social del espacio, particularmente dimensiones relacionadas con vivienda, servicios, economía, derechos sociales y condiciones ambientales. El índice se calculó aplicando agregación lineal de diversos indicadores ponderados. Los pesos se calcularon a través del análisis de componentes principales. Los resultados permiten comprender condiciones de la división social del espacio y establecen una tipología de las formaciones socioterritoriales que coexisten en el área metropolitana. El trabajo aspira a contribuir en el ejercicio del derecho a la ciudad y el territorio en el DMQ.

PALABRAS CLAVE | áreas metropolitanas, desigualdad social, distribución espacial.

ABsTract | We explore the quality of life in the Metropolitan District of Quito (MDQ) by calculating a multidimensional index for the 65 parishes of the MDQ. The index synthetizes determinants of the social division of space, particularly dimensions related to household conditions, access to services, economy, social rights and environmental conditions. The index was calculated by a weighted linear aggregation of indicators. The weights were calculated using principal component analysis. The results obtained support the understanding of the conditions of the social division of the space and establish a typology of social-territorial areas that coexist in the $M D Q$. Our work aims contribute to the perspective of the right to the city and the right to the territory in the MDQ.

KEYWORDs | metropolitan areas, social inequality, spatial distribution. 


\section{Introducción}

\section{Derechos y calidad de vida}

El horizonte axiológico que inspira este trabajo es el ejercicio del derecho a la ciudad y la construcción de una ciudad justa. La idea lefebvriana de un derecho que suponga la apropiación colectiva de la urbe, más allá del acceso al equipamiento urbano, a vivienda digna y a trabajo, coloca el debate en el campo de las relaciones de poder en la ciudad (Ugalde, 2015). La Carta Mundial del Derecho a la Ciudad define el derecho a la ciudad como "el usufructo equitativo de las ciudades dentro de los principios de sustentabilidad, democracia, equidad y justicia social” (Habitat International Coalition [HIC], 2012, p. 185), el cual está fundamentado en tres pilares: el ejercicio pleno de la ciudadanía y, por lo mismo, de todos los derechos humanos que aseguran el bienestar colectivo de los habitantes; la gestión democrática de la ciudad, a través de la participación de la sociedad de forma directa y participativa; y la función social de la propiedad y de la ciudad, fundada en el bien común (Mathivet, 2011). Se trata de un derecho colectivo, cuyas condiciones de producción y ejercicio se construyen socialmente a través de una ciudad justa, como aquella basada en los principios de equidad, democracia y diversidad (Fainstein, 2013).

El abordaje de la dimensión urbana y del derecho a la ciudad no se limita a aquellos espacios con características de alta densidad y aglomeración, sino que se hace extensivo a la noción de territorio. El derecho a la ciudad rebasa los límites de la ciudad y se extiende a las diversas y heterogéneas formas socioterritoriales existentes, a condición de que se cumplan sus postulados básicos; es decir, el usufructo equitativo de los bienes públicos, el mandato de construcción colectiva y participativa de los asuntos de ciudad y el goce efectivo de los derechos humanos. Son varios los autores y colectivos que reivindican una versión integradora e inclusiva del derecho a la ciudad y al territorio; entre ellos, Borja (2012), Correa (2010), Limonad y Monte-Mór (2012).

En cualquier caso, el ejercicio del derecho a la ciudad tiene una doble dimensión. Por un lado, el cumplimento efectivo de esos derechos y el usufructo equitativo de la ciudad; y, por otro, el grado de participación social y política de los ciudadanos, la calidad de la democracia, y la acción colectiva y social. La noción de calidad de vida alude especialmente al acceso y disposición de los satisfactores que permiten cubrir las necesidades fundamentales de los individuos, grupos sociales y comunidades que habitan en la ciudad (Max-Neef et al., 1986). Desde una perspectiva amplia y general, las necesidades de subsistencia, nutrición, protección, seguridad y autorrealización son universales, pero hay pautas culturales y condiciones socioeconómicas que las modelan y las hacen específicas en tiempo y lugar (Lindenboim et al., 2000).

La calidad de vida está constituida por múltiples dimensiones de la vida social a través de las cuales se establece la correspondencia $-\mathrm{O}$ no correspondenciaentre las necesidades objetivas y las demandas subjetivas relativas a la vida social, así como las potencialidades, condiciones y niveles relativos de acceso a su satisfacción (Lindenboim et al., 2000). Esto implica un esfuerzo de reflexión permanente sobre las determinantes de la reproducción de la sociedad urbana en América Latina y sus especificidades históricas y culturales. Los procesos de división del espacio urbano 
no se producen ni explican en ciclos cortos. Por el contrario, se conforman por la concurrencia de largos y reiterados factores de exclusión, empobrecimiento y pérdida de oportunidades de vida para las personas (Katzman \& Retamoso, 2005). La espacialización de las diferencias sociales implica la recurrencia de múltiples factores que se refuerzan, conformando un círculo vicioso. Visualizarlo supone, entonces, mostrar tanto las reiteraciones históricas como las oportunidades de futuro que se dibujan en la realidad presente.

Arriagada (2000) destaca tres condiciones que afectan con severidad a los pobres de los grandes centros urbanos, influyendo con especial fuerza en su capital social y físico: i) la segregación y el mercado de suelo urbano; ii) la desigualdad de acceso a la infraestructura y equipamiento urbano (activos físicos públicos de la ciudad); y iii) la debilidad de las finanzas y capacidad de inversión de los municipios que concentran a los pobres. Además de estas determinantes, la pobreza y la vulnerabilidad urbana están vigorosamente relacionadas con las formas de relación laboral y sus implicaciones en los hogares; de manera particular, con la calidad del empleo, como también con su estabilidad y productividad, factores generados por la concurrencia de mercados laborales limitados y estructuras económicas poco diversificadas, así como con fuerza de trabajo menos cualificada y menores tasas de inversión privada (Polèse, 1998).

La estrategia de sobrevivencia de los hogares incluye, en muchos casos, el trabajo infantil, la sobrecarga laboral y otras formas extremas de precarización. Se reproducen así dinámicas de exclusión económica que restringen las posibilidades de las personas de acceder a los medios necesarios para participar en el consumo y producción. La exclusión económica se acompańa de exclusión política, caracterizada por la constante marginación de los circuitos de toma de decisiones; y, finalmente, de exclusión cultural, expresada en la precariedad de las redes sociales primarias de los más pobres, e incluso en la estigmatización social y espacial. Como es lógico, estas tres formas de exclusión interactúan entre sí, produciendo un círculo perverso de perpetuación de las inequidades (Arriagada, 2000).

El acceso desigual de la población a una vivienda digna o a un espacio público adecuado ha sido explicado fundamentalmente por el precio del suelo como principal mecanismo de segregación residencial (Sabatini \& Brain, 2008); a ello se suman otros factores, como la estructuración de una demanda que incorpora aspectos aspiracionales y de distinción social, así como la permisividad del marco normativo local (Portes \& Roberts, 2008; Roberts \& Portes, 2008; Sabatini, 2003; Sabatini \& Brain, 2008). Además de estas tradicionales restricciones, en las sociedades contemporáneas adquiere un rol decisivo en la calidad de vida el acceso a las tecnologías digitales de información y conocimiento. La calidad de ese acceso depende de un conjunto de dimensiones tecnológicas, de telecomunicaciones, económica, de información y educación, lo que le da un carácter transversal y múltiple, tanto por sus determinantes como por sus efectos (Chaparro Mendivelso, 2007, 2010).

El acceso a bienes públicos y el ejercicio de derechos como la salud, la educación y la seguridad son definitivos en la creación de oportunidades para el desarrollo de un ser humano. Su carencia provoca una directa erosión de la calidad de vida, 
desembocando no solo en desigualdad estática, sino también en su perpetuación y profundización en el largo plazo (Arriagada, 2000). De hecho, los mecanismos y procesos de exclusión originan que los hogares tengan un riesgo diferencial que segmenta personas y hogares en función de su condición actual y de su capacidad de respuesta, produciendo un cierto grado de vulnerabilidad. De manera particular, los trabajadores urbanos que dependen exclusivamente de los ingresos provenientes del empleo o de los precarios ingresos de la economía informal, viven expuestos a esa condición de vulnerabilidad social (Pizarro, 2001).

Asimismo, el ámbito de la calidad ambiental urbana ha adquirido durante los últimos años una gran relevancia y se ha dado un activo proceso de producción y análisis de indicadores ambientales relativos a la morfología urbana, a la complejidad, el metabolismo urbano y la biodiversidad, entre otros aspectos (Escobar, 2006; Henríquez, 2004; Rueda, 2008). El principal límite con que se topa el análisis de esta dimensión es la pobre estadística ambiental en el nivel de unidades suburbanas. Suele existir información relativa a algunos aspectos del metabolismo urbano (recolección de desechos, uso de combustible), pero es muy limitada en aspectos como la morfología o la calidad del uso del espacio público. En cualquier caso, se trata de un componente sustantivo en el análisis de la calidad de vida contemporánea.

Ahora bien, el ejercicio diferenciado de los derechos y de la calidad de vida al que se ha hecho referencia se expresa en términos espaciales en un patrón de estructuración territorial característico. Aunque habría que matizar que la estructuración espacial no es un reflejo automático de la estructura social (Bourdieu, 2000), hay una clara correlación en la distribución de oportunidades de acceso a los bienes materiales y simbólicos ofrecidos por la ciudad en función de variables como la estratificación socioeconómica, nivel educativo, ingresos, categoría socioocupacional, condiciones materiales de vida, entre otras (Molinatti, 2013).

Esa compleja relación de formas espaciales y procesos socioeconómicos no es simple ni unívoca. La calidad de vida se objetiviza, adquiere concreción y materialidad en el acceso a la ciudad como macrobien público, en el sentido más amplio (vivienda, servicios, infraestructura), pero, al mismo tiempo, el espacio urbano actúa como una dimensión constitutiva de la vida social, condicionando la reproducción de las desigualdades (Segura, 2014). Por otro lado, la localización residencial de las familias está condicionada por el complejo proceso de reorganización espacial de las ciudades, impulsado en el contexto de una globalización subordinada (De Mattos, 2010).

La noción de división social del espacio residencial (DSER) propuesta por Duhau (2013) expresa las formas espaciales que adopta la distribución residencial de los distintos estratos socioeconómicos en una aglomeración urbana. Teniendo un carácter más comprehensivo, tal categoría permite mayor amplitud en la construcción de las variables sobre las que se pretende identificar el patrón socioespacial. Se trata de la "consecuencia" de las múltiples dimensiones que han producido el espacio en la ciudad a lo largo de la historia. En esta línea argumental, Duhau entiende como estructura socioespacial al conjunto de formas espaciales específicas y discernibles que adopta la DSER (Duhau, 2013); vale decir, la combinación particular de tipos o patrones espaciales que permiten mapear la calidad de vida. 
El análisis de la DSER supone la definición de unidades o subdivisiones territoriales. En general se entendería que mientras más pequeña es esa unidad, mayor sería el grado de homogeneidad. Para el caso del análisis realizado en este estudio se adoptan las unidades parroquiales, que tienen un doble carácter a nivel urbano y rural y que, además, en el caso de Ecuador, son las unidades territoriales mínimas con competencias administrativas y de gestión pública.

Una arista ineludible del análisis es la dicotomía urbano/rural. Este trabajo se aproxima a la idea de que "el medio urbano deja de ser el espacio de la ciudad para transformarse en el espacio social total... una especie de metáfora para la comprensión del espacio social contemporáneo" (Limonad \& Monte-Mór, 2012). Hay un indudable proceso de urbanización de toda la sociedad, incluyendo remotos y pequeños poblados que levantan demandas y necesidades de calidad de servicios y empleo; pero, sobre todo, porque la expansión del capitalismo ha resignificado aceleradamente los territorios, imponiendo formas diversas de modernización, que integran o excluyen a las antiguas zonas rurales, cuya dinámica no puede ser entendida sino como parte de una de carácter global. Este proceso ha implicado la redefinición funcional de las formas, un redimensionamiento de las funciones y una resignificación de los contenidos (Limonad \& Monte-Mór, 2012).

A lo anterior hay que sumar la realidad de la gestión pública en América Latina. Los gobiernos locales tienen a su cargo amplios territorios compuestos por un variado mosaico territorial, que va desde núcleos urbanos consolidados hasta espacios que podrían calificarse como rurales. En el caso del Distrito Metropolitano de Quito (DMQ), coexisten el gobierno municipal, que se enfoca principalmente en el área urbana del distrito, la ciudad de Quito, mientras que las parroquias rurales poseen gobiernos autónomos, con competencias específicas. Esto introduce la dimensión política y social del histórico clivaje urbano-rural, cuyo tratamiento debería incluir políticas públicas específicas que incluyan mecanismos e instrumentos de inversión, gestión y seguimiento que den cuenta de la diversidad socioterritorial.

\section{Mediciones de la calidad de vida}

Hay un extenso trabajo de construcción de índices urbanos. El índice de prosperidad urbana (onU-Habitat, 2010, 2016) se convirtió en una referencia estándar de la situación de las ciudades a nivel global. Pese a los debates alrededor de la noción de "prosperidad urbana" y a la heterogeneidad y limitación de las fuentes de información, algunos centenares de ciudades desarrollaron este ejercicio en las distintas modalidades del índice. Sin embargo, una limitante de este índice es el muy elevado número de indicadores usados y la complejidad de los métodos de ponderación aplicados, lo que puede volver muy abstracta y general la interpretación del valor final del índice, y también puede dificultar la medición sostenida del índice a través de los ańos.

Otros estudios han abordado el análisis de la estructura urbana a partir de variables económicas que indagan si las áreas metropolitanas mantienen los clásicos modelos monocéntricos o tienden a modelos dispersos o híbridos (Rojas Quezada et al., 2009). El presente trabajo se inscribe en aquellos que han abordado la calidad de vida y división espacial en ciudades latinoamericanas (Cabello Quiñones, 2012; 
Consejo Nacional de Desarrollo Urbano [CNDu], 2017; Delfim \& Martins, 2002; González García, 2013; Jiménez Barbosa \& González Borrero, 2014; Leva, 2005; Lindenboim et al., 2000; Tello Aragay, 1999; Vicuńa et al., 2019). Como otro ejemplo, desde la acción pública, el gobierno de Chile ha desarrollado un sistema de indicadores urbanos como una propuesta integrada que permite evaluar el desarrollo urbano y la calidad de vida. La definición de algunos compromisos ha permitido la orientación programática de qué medir, mientras la articulación con el sistema censal permite la generación de indicadores estandarizados (CNDU, 2017).

El trabajo sobre calidad de vida e integración urbana en zonas metropolitanas de Chile desarrollado por Vicuńa et al. (2019) postula que en la relación entre integración urbana y calidad de vida en contextos metropolitanos persiste un crecimiento fragmentado, el cual reproduce viejas y nuevas formas de segregación, impidiendo la integración social. Desde este enfoque se ha analizado la relación entre integración urbana y calidad de vida. La autora operacionaliza la calidad de vida en tres dimensiones clave: condiciones socioculturales, conectividad/movilidad, y vivienda/ entorno. En la primera indaga sobre los procesos de participación en organizaciones sociales, la violencia intrafamiliar, la tasa de embarazo y el desempeńo educativo. La dimensión de vivienda y entorno alude al acceso a servicios y equipamientos urbanos, en especial de plazas y parques, esenciales para la interacción social, ya que permiten la presencia simultánea de distintos grupos sociales. El presente trabajo otorga particular importancia a las dimensión de conectividad y movilidad como factores determinantes en la producción de la "segregación funcional" a partir de la movilidad cotidiana (Avellaneda, 2007) y que afecta de forma diferenciada a las personas (Linares-García et al., 2018).

En Porto Alegre se analizó la calidad de vida a través de cuatro dimensiones. La primera, relativa a las condiciones ambientales, engloba aspectos naturales y físicos de la ciudad: recursos ambientales como el agua, áreas verdes, y gestión de residuos. La segunda remite a las condiciones materiales colectivas relativas al equipamiento e infraestructuras, que incluyen cultura, deportes, ocio, salud, asistencia social, transporte y servicios. La tercera dimensión aborda las condiciones económicas, y pretende analizar la ciudad como núcleo de la actividad económica y de implicaciones individuales, como el consumo y el mercado de trabajo. La cuarta dimensión, definida como sociedad, integra los indicadores relativos a la dimensión social de la ciudad, las relaciones entre las personas, su nivel de participación y pertenencia sociocultural (Delfim \& Martins, 2002).

En el caso del DMQ, el Instituto de la Ciudad (2015) desarrolló un índice de calidad de vida, definido como una herramienta estadística multidisciplinar para el análisis integral y comparativo de la calidad de vida. El índice se compone de 13 dimensiones cuyos resultados se presentan de manera consolidada. Los indicadores y las dimensiones y el índice global se calcularon en una escala entre 0 y 100 , y se realizó una equiponderación que arroja resultados que van desde un índice de 73 en Ińaquito, a 54 en Lloa. En el presente estudio seguimos esta línea de trabajo, proponiendo un índice de calidad de vida que pueda ser aplicado al DMQ y que use indicadores oficiales. La siguiente sección explica los métodos aplicados. 


\section{Métodos}

\section{Área de estudio}

El DMQ es la jurisdicción territorial especial administrada por el municipio del mismo nombre como gobierno autónomo descentralizado. Tiene una población cercana a los 2,8 millones de habitantes, de los cuales alrededor del $68,7 \%$ reside en el área urbana.

El territorio del DMQ abarca alrededor 400 mil hectáreas de topografía irregular, con 44 pisos climáticos y ecosistemas (Municipio del Distrito Metropolitano de Quito [MDMQ], 2012). El Distrito incluye el área urbana consolidada, la ciudad de Quito, que posee una forma alargada localizada entre el volcán Pichincha (oeste) y los valles de Tumbaco y Los Chillos (este). La topografía del DMQ posee una marcada variación altitudinal, donde los valles poseen una altura de alrededor de $2.500 \mathrm{msnm}$, mientras que la ciudad de Quito se encuentra a una altura de $2.850 \mathrm{msnm}$. Hacia el oeste, el volcán Pichincha es en realidad un macizo de varias cumbres con una altura de $4.794 \mathrm{msnm}$. El noroccidente del DMQ posee una altura de alrededor de 1.200 msnm, y cuenta con una importante extensión de bosques tropicales.

En los últimos años, el ritmo de crecimiento demográfico ha sido mayor en la periferia urbana y en los valles colindantes que en la ciudad central, generando con ello un proceso de conurbación y expansión urbana. El DMQ contempla, además, una extensa área rural muy heterogénea, con territorios que van desde espacios conurbados o funcionalmente ligados a las dinámicas centrales, hasta otros propiamente rurales y periféricos, que pueden incluir grandes extensiones de bosques.

Este amplio territorio está gobernado por el Municipio del Distrito Metropolitano, cuyas competencias rigen sin diferenciación en las 65 parroquias en que se organiza, 32 urbanas y 33 rurales. En la legislación ecuatoriana las parroquias rurales están regidas por Gobiernos Autónomos Descentralizados (GAD), que, aunque hacen parte del Distrito Metropolitano, tienen algunas competencias propias relacionadas con la participación y gestión de equipamientos locales. Las parroquias urbanas, en cambio, no tienen representación político-administrativa, pero se consideran unidades de planificación y, en algunos casos, de asignación de recursos. La "ciudad" de Quito no tiene una forma de gobierno específico que no sea el mismo municipio del Distrito. En cualquier caso, el análisis a partir de estas unidades espaciales tiene un valor explicativo y práctico, en la medida en que competencias básicas de prestación de servicios básicos, ordenamiento del suelo y promoción de algunas actividades económicas son responsabilidad municipal.

La Figura 1 muestra el área de estudio, las parroquias del DMQ. Las parroquias urbanas son las de menor tamaño y la mayoría de ellas aparecen enumeradas, indicándose su respectivo nombre al lado izquierdo de la figura, según el número inscrito en el mapa. 
FIgURA I | Área de estudio: el Distrito Metropolitano de Quito (DMQ) y sus parroquias

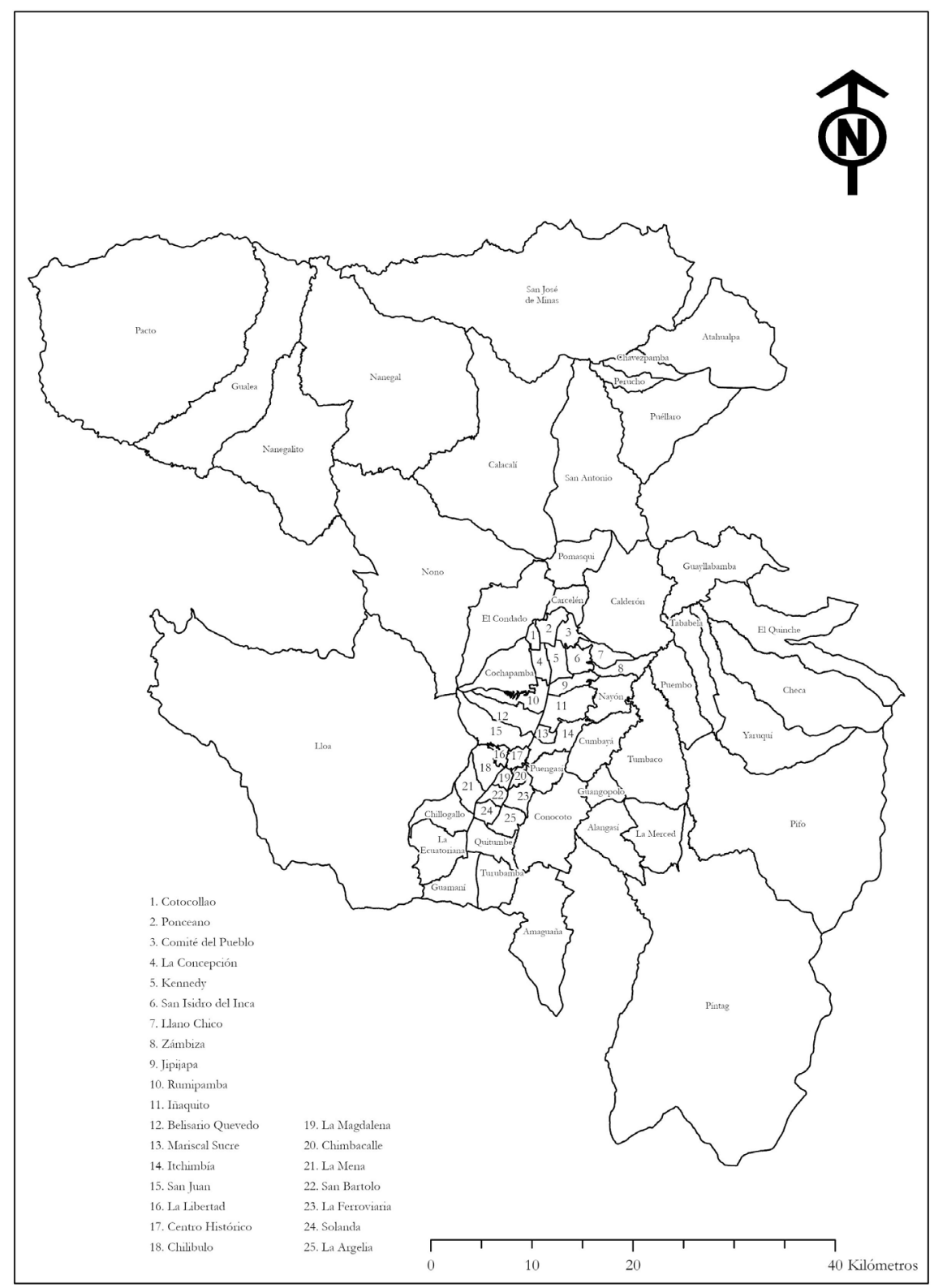

FUENTE: ELABORACIÓN PROPIA, CON BASE EN DATOS ABIERTOS DEL MUNICIPIO DEL DMQ, HTTP://GOBIERNOABIERTO.QUITO.GOB.EC/?PAGE_ID=I I 22 
Esta conformación espacial se ha ido produciendo a lo largo de la historia, pero de manera muy acelerada en el último medio siglo, en que la ciudad decuplicó su población y pasó de ser un núcleo más o menos compacto a tener un crecimiento difuso hacia los extremos norte y sur y hacia los valles oriental y suroriental. En ese camino se ha producido la conurbación con varias localidades que están integradas a la dinámica urbana.

\section{Materiales y métodos}

La información usada proviene del Instituto Nacional de Estadísticas y Censos, a través del Censo de Población y Vivienda del Ecuador del año 2010, y las encuestas de condiciones de vida de 2013-2014; los datos de homicidios provienen de información de la Fiscalía, de 2012, y la superficie de áreas verdes proviene de datos del Municipio del DMQ del año 2013. La escala de análisis del presente estudio es el nivel parroquial, es decir, todos los indicadores usados están expresados a esa escala.

El índice desarrollado posee cuatro dimensiones de calidad de vida. La primera dimensión se denomina hábitat, vivienda digna y servicios básicos, y se conforma de los siguientes indicadores: 1) porcentaje de hogares que disponen de computador (c); 2) porcentaje de hogares con acceso a internet (I);3) porcentaje de hogares con acceso a la red pública de agua (A); 4) porcentaje de hogares con vivienda propia $(\mathrm{VP}) ; 5)$ porcentaje de hogares con acceso a red pública de alcantarillado (AL); 6) porcentaje de viviendas con adecuados materiales (AM); y 7) porcentaje de hogares sin hacinamiento (SINH).

La segunda dimensión representa calidad de la inserción económica, pobreza, equidad, capacidad productiva y ocupación. Incluye los indicadores de: 1) porcentaje de trabajo infantil (TI); 2) porcentaje de pobres por consumo (PC); 3) coeficiente de Gini (CG); y 4) porcentaje de población económicamente activa (PEA). Estos indicadores también fueron obtenidos de datos censales y estructurados por el Centro de Investigaciones de Políticas Públicas y Territorio, de flacso (CITEFLACSO, 2015). El coeficiente de Gini fue multiplicado por 100 para homogenizar la variable a porcentaje. Al crear índices, fue necesario normalizar variables antes de sumarlas (Cabrera-Barona et al., 2015; Cabrera-Barona et al., 2016). En este estudio se aplicó una normalización de 0 a 100, donde 0 es el valor más bajo y 100 el valor más alto de un indicador.

La tercera dimensión se denomina dimensión de derechos sociales y seguridad pública. Esta dimensión incluye: 1) porcentaje de asistencia a programas de desarrollo infantil (DI); 2) porcentaje de asistencia a educación secundaria (ES); 3) porcentaje de asistencia a educación superior (ESU); 4) porcentaje de analfabetismo (AN); 5) porcentaje de trabajadores con seguridad social (TSS); 6) porcentaje de adultos mayores con seguridad social (AMSs); y 7) número de homicidios (H). Este último indicador se normalizó mediante la técnica máximo-mínimo y multiplicando este resultado por 100 .

La cuarta dimensión se relaciona al tema ecosistémico, e incluye consumo energético, desechos y áreas verdes públicas. Específicamente, sus indicadores son: 1) porcentaje de viviendas que eliminan basura por sistema de recolección de desechos $(\mathrm{RD})$; 2) nivel de consumo energético $(\mathrm{CH})$; y 3$)$ superficie de áreas verdes públicas 
en metros cuadrados (Av). Este último indicador se normalizó mediante la técnica máximo-mínimo y multiplicando este resultado por 100 .

La Tabla 1 resume los indicadores usados para conformar cada dimensión.

TABLA I Indicadores para construir el índice de calidad de vida

\begin{tabular}{|c|c|}
\hline DIMENSIONES & INDICADORES \\
\hline \multirow{7}{*}{ D1 } & C: porcentaje de hogares que disponen de computador \\
\hline & I: porcentaje de hogares con acceso a internet \\
\hline & A: porcentaje de hogares con acceso a la red pública de agua \\
\hline & VP: porcentaje de hogares con vivienda propia \\
\hline & AL: porcentaje de hogares con acceso a red pública de alcantarillado \\
\hline & AM: porcentaje de viviendas con adecuados materiales \\
\hline & SINH: porcentaje de hogares sin hacinamiento \\
\hline \multirow{4}{*}{ D2 } & TI: porcentaje de trabajo infantil \\
\hline & PC: porcentaje de pobres por consumo \\
\hline & CG: coeficiente de Gini \\
\hline & PEA: porcentaje de población económicamente activa \\
\hline \multirow{7}{*}{ D3 } & DI: porcentaje de asistencia a programas de desarrollo infantil \\
\hline & ESU: porcentaje de asistencia a educación superior \\
\hline & ES: porcentaje de asistencia a educación secundaria \\
\hline & AN: porcentaje de analfabetismo \\
\hline & TSS: porcentaje de trabajadores con seguridad social \\
\hline & AMSS: porcentaje de adultos mayores con seguridad social \\
\hline & H: número de homicidios \\
\hline \multirow{3}{*}{ D4 } & $\begin{array}{l}\text { RD: porcentaje de viviendas que eliminan basura por sistema de recolección de } \\
\text { desechos }\end{array}$ \\
\hline & $\mathrm{CH}$ : nivel de consumo energético \\
\hline & AV: superficie de áreas verdes públicas en metros cuadrados \\
\hline
\end{tabular}

FUENTE: ELABORACIÓN PROPIA

El índice de calidad de vida se calcula con la sumatoria ponderada de todas las cuatro dimensiones:

$$
\mathrm{CV}=\mathrm{W}_{\mathrm{D} 1} \mathrm{D}_{1}+\mathrm{W}_{\mathrm{D} 2} \mathrm{D}_{2}+\mathrm{W}_{\mathrm{D} 3} \mathrm{D}_{3}+\mathrm{W}_{\mathrm{D} 4} \mathrm{D}_{4}
$$

En donde $\mathrm{D}_{1}, \mathrm{D}_{2}, \mathrm{D}_{3}, \mathrm{y} \mathrm{D}_{4}$ son las dimensiones y las $\mathrm{W}$ representa los pesos para cada una de ellas.

La $\mathrm{D}_{1}$ se calcula de la siguiente manera:

$$
\mathrm{D}_{1}=\mathrm{W}_{\mathrm{C}} \mathrm{C}+\mathrm{W}_{1} \mathrm{I}+\mathrm{W}_{\mathrm{A}} \mathrm{A}+\mathrm{W}_{\mathrm{VP}} \mathrm{VP}+\mathrm{W}_{\mathrm{AL}} \mathrm{AL}+\mathrm{W}_{\mathrm{AM}} \mathrm{AM}+\mathrm{W}_{\mathrm{SINH}} \mathrm{SINH}
$$

$\mathrm{La} \mathrm{D}_{2}$ se calcula con la fórmula:

$$
\mathrm{D}_{2}=\mathrm{W}_{\mathrm{PEA}} \mathrm{PEA}-\mathrm{W}_{\mathrm{CG}} \mathrm{CG}-\mathrm{W}_{\mathrm{PC}} \mathrm{PC}-\mathrm{W}_{\mathrm{TI}} \mathrm{TI}
$$

donde las W representan los pesos de cada uno de los indicadores descritos anteriormente. 
$\mathrm{La} \mathrm{D}_{3}$ :

$$
\mathrm{D}_{3}=\mathrm{W}_{\mathrm{DI}} \mathrm{DI}+\mathrm{W}_{\mathrm{ES}} \mathrm{ES}+\mathrm{W}_{\mathrm{ESU}} \mathrm{ESU}_{\mathrm{AN}} \mathrm{AN}+\mathrm{W}_{\mathrm{TSS}} \mathrm{TSS}+\mathrm{W}_{\mathrm{AMSS}} \mathrm{AMSS}-\mathrm{W}_{\mathrm{H}} \mathrm{H}
$$

$\mathrm{La} \mathrm{D}_{4}$ :

$$
\mathrm{D}_{4}=\mathrm{W}_{\mathrm{RD}} \mathrm{RD}+\mathrm{W}_{\mathrm{CH}} \mathrm{CH}+\mathrm{W}_{\mathrm{AV}} \mathrm{AV}
$$

Las dimensiones se normalizaron utilizando la técnica máximo-mínimo antes de la sumatoria ponderada. Las W de todos los indicadores o dimensiones representan los pesos de estos. Tanto los pesos de los indicadores como los pesos de las dimensiones fueron calculados mediante la aplicación de análisis de componentes principales (Organisation for Economic Co-operation and Development [oECD], 2008). Primero se obtiene la matriz de componentes rotada. Luego se calcula el cuadrado de la matriz rotada y después se normaliza este resultado. Se extraen los valores mayores de componentes principales de esta matriz normalizada, para cada indicador. Se calculan también las sumatorias normalizadas de cada fila de matriz que fue elevada al cuadrado. El siguiente paso es multiplicar estas sumatorias normalizadas para los valores de componentes principales de cada indicador extraídos anteriormente, lo que da como resultado una nueva matriz de pesos. Los pesos finales de cada indicador (o dimensión, de ser el caso) se obtienen a partir de la normalización de esta matriz de pesos.

\section{Resultados}

La Tabla 2 muestra la media y desviación estándar de las dimensiones y del índice de calidad de vida en el DMQ. Todas las medidas están estandarizadas en el rango de 0 a 100, donde 100 significa calidad de vida más alta y 0 calidad de vida más baja en la dimensión o en el índice final. Se aprecia que la dimensión con la media más alta es la dimensión 4 (consumo energético, desechos, y áreas verdes públicas), y la dimensión con la media más baja es la dimensión 1 (servicios básicos, vivienda digna y hábitat). En la dimensión 1, gran parte de las parroquias poseen valores de entre 30 y 60, y el valor de 0 se obtuvo para la parroquia Gualea, mientras que el valor de 100 se dio para la parroquia Rumipamba. La dimensión 2 (calidad de la inserción económica, pobreza, equidad, capacidad productiva y ocupación) posee una media de 64,86, y una gran parte de las parroquias poseen valores de entre 60 a 80 en esta dimensión. La parroquia con valor mínimo en la dimensión 2 es San José de Minas y la que posee el máximo valor es la parroquia Mariscal Sucre. Finalmente, la dimensión 3 (derechos sociales, calidad de vida y seguridad pública) posee una media de 54,93; la parroquia con menor calidad de vida es Nono, y la que tiene mayor calidad de vida es Rumipamba. Hay muchas parroquias que poseen valores de entre 40 a 70 en esta dimensión. En la dimensión 4, el valor de 0 es para la parroquia San José de Minas, y el valor 100 para la parroquia Ińaquito. En la dimensión 4, la mayoría de las parroquias poseen valores de entre 70 a 90. Las desviaciones estándar muestran que los datos no poseen una dispersión extrema respecto a la media. Los intervalos de confianza muestran cuánto podrían variar las medias en función de las desviaciones estándar y del tamaño de muestra (65). Por 
ejemplo, en el caso de la dimensión 1, los intervalos de confianza son de $\pm 6,20$, por lo que la media de la dimensión podría tomar valores de entre 40,44 a 52,84. En el caso del índice final de calidad de vida (la suma ponderada de las cuatro dimensiones), el valor promedio es de 58,71, lo que indica que el DMQ, visto de manera general, posee un cumplimiento regular de todos los derechos considerados. El valor mínimo de índice final es 4,50 (parroquia San José de Minas) y el máximo es 95,80 (parroquia Rumipamba).

TABLA 2 Medias y desviaciones estándar de las dimensiones y el índice

\begin{tabular}{|l|c|c|c|}
\hline DIMENSIONES/ÍNDICE & MEDIA & $\begin{array}{c}\text { DESVIACIÓN } \\
\text { ESTÁNDAR }\end{array}$ & $\begin{array}{c}\text { INTERVALOS DE } \\
\text { CONFIANZA }\end{array}$ \\
\hline D1 & 46,64 & 25,14 & $\pm 6,20$ \\
\hline D2 & 64,86 & 23,58 & $\pm 5,82$ \\
\hline D3 & 54,93 & 21,41 & $\pm 5,28$ \\
\hline D4 & 69,31 & 22,82 & $\pm 5,63$ \\
\hline Índice & 58,71 & 21,89 & $\pm 5,41$ \\
\hline
\end{tabular}

FUENTE: ELABORACIÓN PROPIA

La Figura 2 muestra las cuatro dimensiones consideradas expresadas de manera espacial.

La primera dimensión muestra, con más claridad que las siguientes, las brechas entre las zonas urbanas y rurales en el territorio. Las parroquias urbanas cuentan con coberturas de servicios más altas, mejores condiciones de las viviendas y servicios avanzados, como internet. La zona rural posee las tasas más altas de propiedad de la vivienda, pero eso no se corresponde con la calidad de esta. Persisten en zonas rurales grandes déficit de servicios básicos y una gran brecha digital. Aun dentro de las zonas rurales, es posible distinguir diferencias entre las parroquias situadas en el este del DMQ, con las parroquias occidentales. Las primeras cuentan con mayor población, una más larga historia de conformación institucional y mayor integración a ejes como el desarrollo agrícola y el turismo, mientras que las occidentales corresponden a zonas protegidas y agrícolas.

En relación con la segunda dimensión, se observa que el hipercentro del DMQ presenta niveles bajos de privaciones, pero a la vez mayor desigualdad. Es llamativo que el centro histórico y algunos de los barrios inmediatos aparezcan con valores inferiores al centro norte, e incluso a los del sur de la ciudad consolidada. Estos cambios podrían reflejar las dinámicas de empobrecimiento vinculadas al ingreso-consumo, así como al envejecimiento de la población. Hay sectores ricos que presentan un coeficiente de Gini bajo (Ińaquito o Rumipamba), lo cual puede reflejar la homogeneidad de altos ingresos, de trabajos bien remunerados. Las zonas rurales son las más equitativas en la distribución del ingreso en relación con el resto del territorio, considerando, sin embargo, que tienen menor población. No debe dejar de señalarse lo que ocurre con algunos sectores de transición urbano-rural; en el caso de Nayón, por ejemplo, se encuentra la existencia tanto de residencias de personas con altos ingresos como de bajos ingresos. 
FIGURA 2 | Dimensiones del índice de calidad de vida. Las parroquias urbanas poseen calidad de vida más alta que las parroquias rurales considerando las dimensiones calculadas

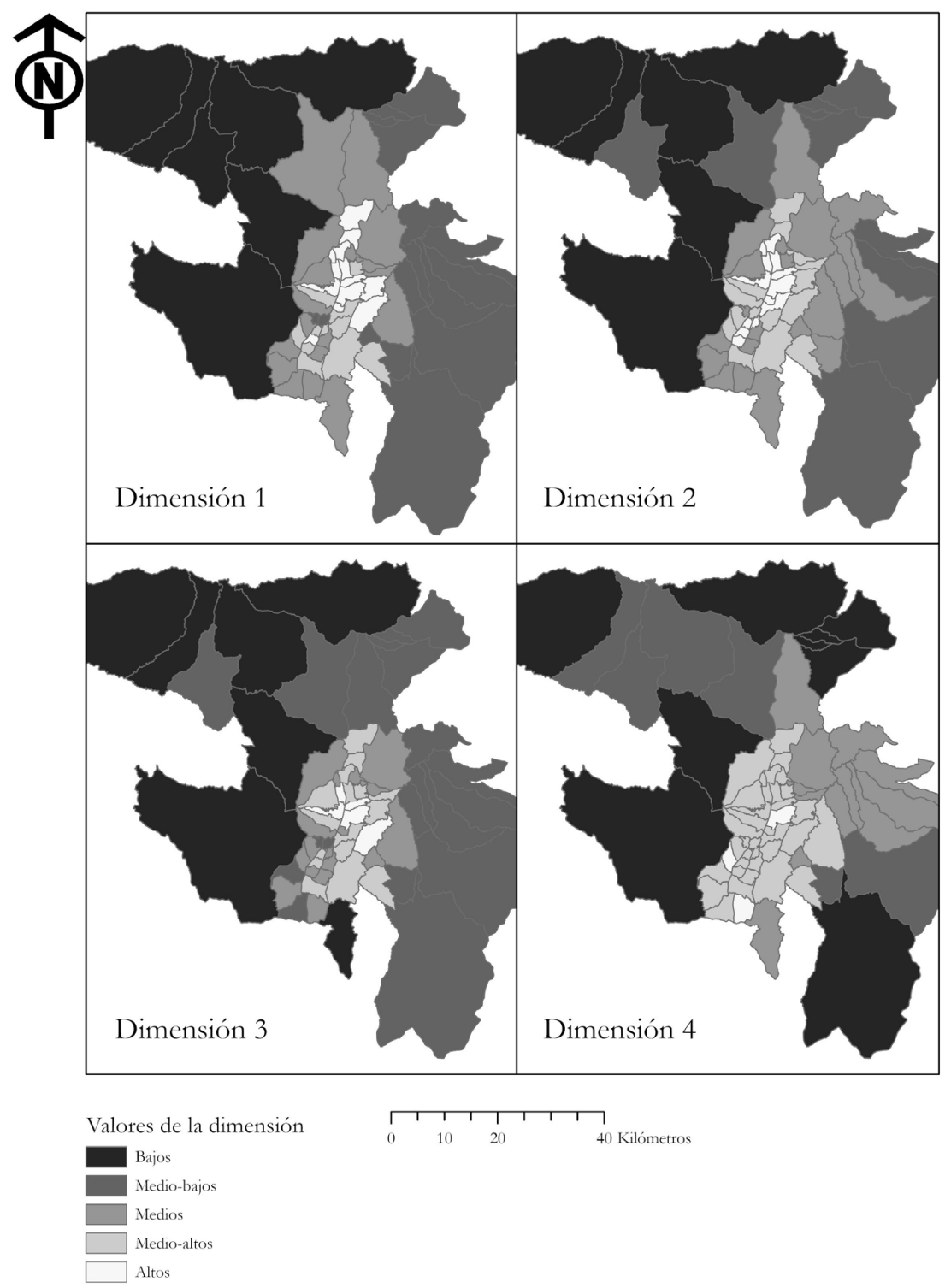


Respecto a la tercera dimensión, se mantiene un patrón similar al primero. En cuanto a la asistencia al nivel secundario de educación, se evidencia claramente una brecha urbano-rural. La asistencia y procedencia de estudiantes universitarios en el DMQ es marcadamente diferente a la de zonas rurales, concentrándose en la zona centro norte y en general en el área urbana. Finalmente, los sectores rurales tienen un menor porcentaje de personas afiliadas a la seguridad social.

La cuarta dimensión, relativa los aspectos ambientales, posee un patrón espacial algo diferente al de las dimensiones anteriores. La zona urbana consolidada y las parroquias más próximas tienen niveles muy similares de recolección de residuos. Los valores del consumo de energía eléctrica se encuentran cercanos a la cobertura total en el conjunto de parroquias. Incluso, el indicador de acceso a espacio público tiene mejores resultados en algunas parroquias de la periferia urbana próximas a equipamientos recreativos de alcance metropolitano.

La Figura 3 presenta el índice de calidad de vida para el DMQ. En general se aprecia un gradiente de mayor a menor calidad de vida y derechos en el gradiente que va desde el consolidado urbano del DMQ hacia sus parroquias rurales. Las parroquias rurales con menores niveles de calidad de vida según el conjunto de indicadores usados para construir el índice son las que se encuentran en el norte y occidente del DMQ. Sin embargo, es necesario tomar en cuenta que estas parroquias constituyen territorios muy rurales, con extensas coberturas de suelo naturales, por lo que se debe ser cautelosos al catalogar uno u otro territorio como de "menor calidad de vida”. Aunque estas parroquias pueden presentar mayor vulnerabilidad social y económica, las mismas podrían ofrecer a sus habitantes aspectos positivos de calidad de vida, como mejor acceso a productos agrícolas locales de calidad. Las parroquias rurales del este del DMQ están en mejores condiciones, sobre todo aquellas que se encuentran adyacentes a las parroquias urbanas. En general, los resultados obtenidos sugieren menor acceso a servicios en las parroquias rurales, corroborando situaciones de inequidad según las cuales lo rural se encuentra marginalizado en materia de procesos de desarrollo y aplicación de políticas públicas para mejoramiento de la calidad de vida. 
FIGURA 3 | Índice de calidad de vida para el DMQ

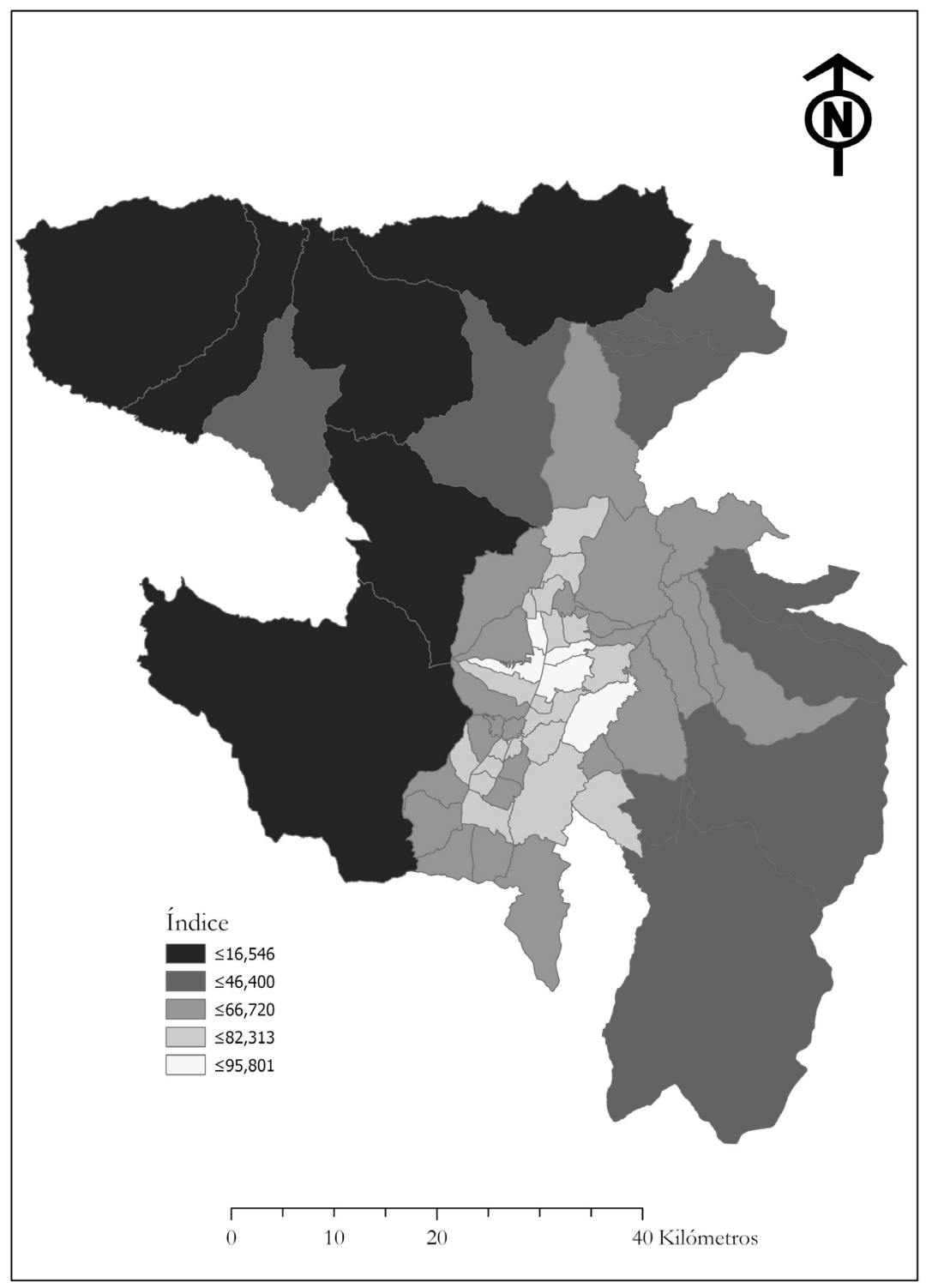

FUENTE: ELABORACIÓN PROPIA 


\section{Discusión}

Los resultados del índice de calidad de vida aplicado a las 65 parroquias arrojan una estructura socioespacial heterogénea. Se trata de un patrón espacial diferenciado para el área del Distrito Metropolitano de Quito, en el que la más evidente característica es la divergencia entre la zona urbana y su inmediata periferia, frente al amplio territorio de las zonas rurales.

En efecto, la Figura 3 muestra cinco tipologías o grupos de parroquias según el índice de calidad de vida. El primer tipo se concentra en las parroquias urbanas de Rumipamba, Ińaquito, Jipijapa, Mariscal Sucre y Concepción. Las cinco parroquias urbanas conforman un continuum territorial localizado en el centro-norte financiero y residencial de la ciudad. Además de coberturas universales de servicios básicos, disfrutan de indicadores superiores a la media en aspectos como tenencia de computador y acceso a internet y son consistentes en mejores resultados en materia de menor pobreza, mejor nivel de instrucción, mayor escolaridad. Este núcleo se extiende hacia la parroquia rural de Cumbayá, que, conurbada, se ha convertido en la principal zona de residencia de los sectores de ingresos altos. Este es el espacio de mayor concentración de la actividad comercial, financiera y de servicios.

En el segundo tipo se encuentran veinte parroquias que conforman en su mayor parte la ciudad compacta, producto de la expansión urbana desde la segunda mitad del siglo xx. Se trata de barrios y condominios consolidados y regularizados con altas densidades en relación con el área metropolitana. Dentro de este tipo se pueden identificar, a su vez, tres agrupamientos, dos de los cuales están en el área urbana: las parroquias de la zona urbana centro-sur de la ciudad (San Bartolo, La Magdalena, Solanda, Chimbacalle, La Mena, Itchimbia, Puengasí, Quitumbe, Mariscal Sucre, Belisario Quevedo), así como las de la zona norte (Kennedy, Ponceano, Cotocollao, Carcelén, San Isidro del Inca). Estas dos zonas urbanas configuran un área relativamente continua y compacta de sectores medios y populares, caracterizados por adecuadas coberturas de servicios básicos, pero con segregación digital, mayor nivel de pobreza por consumo y menor nivel educativo que el primer tipo. Finalmente, el segundo tipo incluye algunas parroquias rurales con buena dotación de servicios, cercanas al área urbana consolidada (Nayón, Conocoto, Pomasqui, Alangasí).

El tercer grupo de parroquias tiene un índice equivalente a la media de DMQ. Como muestra la Figura 3, se trata de una extendida periferia de la ciudad, constituida a su vez por barrios y asentamientos populares y parroquias rurales que están en un segundo anillo respecto del área consolidada, conformando dos grandes zonas de expansión en el norte, sur y este. En ciertos casos se trata de asentamientos de las dos últimas oleadas de migración y expansión urbana, que pueden tener déficit de los servicios básicos y de acceso educativo. En este grupo se encuentran parroquias como La Ecuatoriana, Turubamba, Calderón, Comité del Pueblo y El Condado, pero también algunas áreas de más remota constitución, cercanas al centro de la ciudad, que se mantuvieron como zonas de residencia de estratos populares y han experimentado estancamiento o deterioro socioeconómicos. Son los casos de Chilibulo, La Ferroviaria, La Argelia, Chillogallo, Guamaní y, hacia el noroccidente, Cochapamba, donde incluso se encuentra una de las tradicionales comunas 
intraurbanas. Llama la atención que en este grupo se encuentren algunas de las parroquias del núcleo histórico de la ciudad y su anillo inmediato (Centro Histórico, San Juan, La Libertad). Si bien tienen cobertura plena de servicios básicos, la calidad de la vivienda y el sistemático deterioro socioeconómico, ligado al envejecimiento o insuficiencia de equipamientos, ha ido produciendo el empobrecimiento y envejecimiento de la población o directamente el abandono. De hecho, se trata de una zona que ha decrecido demográficamente en los últimos periodos censales.

También se encuentran parroquias rurales muy heterogéneas y en proceso de transición, pero que aún mantienen población con pobreza y carencia de servicios: Tumbaco, Llano Chico, Zámbiza, San Antonio, Tababela, Puembo, Guangopolo. Estas parroquias rurales actualmente experimentan el avance de la frontera urbana y es previsible que se transformarán por la presencia de equipamientos metropolitanos, como el nuevo aeropuerto internacional y la zona logística.

Los tipos cuatro y cinco están constituidos íntegramente por parroquias rurales, lo cual da cuenta de esta fractura socioespacial. El cuarto tipo está compuesto por diez parroquias rurales con pequeños centros o núcleos urbanos, y actividades ligadas a la agricultura. Mantienen un patrón de cabecera parroquial, anejos, barrios y comunas campesinas. Se trata de El Quinche, Calacalí, La Merced, Checa, Pifo, Atahualpa, Chavezpamba, Perucho, Nanegalito, Píntag, Puéllaro.

Finalmente encontramos a la ruralidad periférica, situada en la zona occidental más apartada del distrito y formada por Lloa, Nanegal, Gualea, Pacto, Nono, San José de Minas. En este grupo se ubican las parroquias rurales situadas al oeste del área urbana consolidada y del volcán Pichincha, con amplias coberturas de vegetación natural de alta importancia ecológica, por lo que poseen zonas protegidas. Además, desarrollan actividades agrícolas de productos tropicales de ciclo corto y ganaderos, con núcleos poblados que cumplen funciones de centros regionales. Son las parroquias de menor cantidad y mayor dispersión de población.

Los resultados obtenidos en este trabajo muestran, a partir de la aplicación índice de calidad de vida, que el área metropolitana del DMQ presenta significativos niveles de segregación residencial y socioeconómica. El análisis se ha realizado sobre una matriz territorial basada en el nivel parroquial que, aunque tiene distinta jerarquía a nivel urbano y rural, supone una escala de información y planificación relevantes. Como toda unidad de análisis, se compone de realidades heterogéneas, pero facilita la consolidación y análisis de la información censal.

El DMQ se inscribe en las tendencias generales del proceso difuso y expansivo de las áreas metropolitanas hacia el policentrismo, acompañado de un proceso de suburbanización privada de las elites que contrasta con el incremento del hábitat precario (Ciccolella, 2008). En este caso, la dinámica descrita se ve reflejada en la compleja interrelación entre lo urbano y lo rural. En efecto, en el área urbana consolidada se pueden identificar tres dinámicas socioespaciales: el centro norte financiero y residencial habitado por sectores altos, que presenta valores altos en todas las dimensiones; el gran continuum urbano, que se matiza con las periferias más recientes; y la realidad del deterioro del núcleo histórico central de la ciudad. En el área rural se pueden identificar también tres dinámicas: las parroquias más próximas, que están conurbadas y han sido asimiladas en la lógica de la ciudad 
o están en pleno proceso de transición; las parroquias orientales, con déficit de servicios y pobreza; $y$, finalmente, la abandonada periferia occidental.

Estos resultados responden a los procesos históricos específicos de conformación de la ciudad y a las funciones diferenciadas de cada zona en la dinámica metropolitana (Ludeña, 2006). La estructura obtenida puede asimilarse a las distintas etapas de desarrollo urbano de Quito, muy cercana a los modos de estructuración y funcionamiento (Bähr \& Borsdorf, 2005): la ciudad colonial compacta, la ciudad sectorial, la ciudad polarizada, y finalmente la ciudad fragmentada y dispersa de las últimas décadas.

En esta configuración territorial debe llamarse la atención a la asimetría entre la oferta de servicios y equipamientos, altamente concentrados en el hipercentro y en la zona consolidada, que contrasta con el crecimiento demográfico situado en la periferia (MDMQ, 2012). Esta "paradoja" explica una parte importante de la calidad, coberturas y acceso a servicios y bienes públicos, pero además repercute en la gran cantidad de desplazamientos en la ciudad. El crecimiento de las últimas décadas ha desbordado la capacidad de producción de infraestructura y equipamientos en las zonas más pobres y de mayor crecimiento. No escapa, sin embrago, el hecho de que esta situación refleja las correlaciones sociales y políticas en la matriz de poder local, en las que las zonas rurales y periféricas tienen un menor peso relativo.

El índice de calidad de vida tiene por objetivo mostrar la satisfacción de las necesidades que conciernen al bienestar de los habitantes de las ciudades. La dimensiones e indicadores seleccionados han permitido dar cuenta de un alto nivel de correlación entre los distintos componentes, quizá con excepción de la dimensión ambiental, que debería ser enriquecida con nuevos indicadores. Se confirma, en esta medida, la fuerte correlación entre la calidad del hábitat y servicios, incluyendo ahora la conectividad digital, con el ejercicio de los derechos básicos y la calidad de la inserción en la estructura económica. En términos generales, los indicadores muestran datos razonables de cobertura de varios servicios, pero a la vez una frágil condición económica de la población por la precariedad de los empleos y la volatilidad de los ingresos. Se producen con ello nuevas formas de segregación y desigualdad.

Es preciso destacar que, a partir de este estudio sobre la calidad de vida en el DMQ, se señalan al menos tres desafíos muy claros. Por un lado, la necesidad de incorporar aquellas dimensiones políticas y participativas que sirvan de base para analizar la acción colectiva y las formas de gestión del poder en la ciudad, lo cual permitiría construir una mirada más completa del ejercicio del derecho a la ciudad. El segundo aspecto tiene que ver con las limitaciones en materia de actualidad y amplitud de la data. Buena parte de la información corresponde al censo de 2010, que debía actualizarse el año 2020, pero persiste un déficit en la calidad de los registros administrativos, los mismos que permitirían información actualizada en servicios o coberturas. En un futuro análisis se podría comparar el índice presentado con el mismo índice producido con datos actualizados, como los del nuevo Censo de Población y Vivienda, que se está preparando al momento de escribir el presente artículo y se espera se tengan sus resultados en el año 2021. Es indispensable continuar con nuevas líneas de investigación orientadas a explicar aquellos determinantes que den cuenta de la conformación y evolución de los patrones socioespaciales 
actuales, a fin de detener y revertir los procesos de segregación e inequidad. De manera concreta, los resultados alertan sobre la necesidad de construir enfoques y metodologías adecuados a las realidades rurales y periféricas o formaciones socioterritoriales en acelerados procesos de transición económica y demográfica. También consideramos pertinente que futuras investigaciones puedan enlazar el estudio de los indicadores usados en este trabajo con indicadores de uso y cobertura de suelo, incluyendo áreas protegidas. Adicionalmente, se abre como un campo de estudio prometedor la posibilidad de desarrollar otros índices multidimensionales, usando para ello indicadores no considerados en esta investigación y diferentes métodos para generar pesos de los indicadores.

El diagnóstico presentado aspira a cualificar la formulación de políticas públicas con alto nivel de especificación socioespacial, comprender la sinergia del conjunto de dimensiones que hacen parte de la vida de las personas y priorizar el ejercicio de los derechos de sus habitantes. Creemos que los resultados de este estudio pueden guiar procesos de políticas públicas que prioricen la atención en zonas con mayores privaciones sociales y económicas, y que minimicen las inequidades territoriales dentro del Distrito Metropolitano de Quito. La espacialización de características de territorios a escala subprovincial o subcantonal, como los parroquiales, puede apoyar también la localización efectiva y eficiente de recursos por parte de los gobiernos locales. Todo esto podría equilibrar relaciones de poder de decisión de lo local frente a lo nacional.

\section{Agradecimientos}

Augusto Barrera agradece a la maestría de estudios urbanos de FLACso Ecuador, en donde se realizó esta investigación durante su periodo de docencia.

\section{Referencias bibliográficas}

Arriagada, C. (2000). Pobreza en América Latina: Nuevos escenarios y desafíos de politicas para el hábitat urbano. Serie medio ambiente y desarrollo, 27 [LC/L.1429-P]. Comisión Económica para América Latina y el Caribe (CEPAL). https://repositorio.cepal.org/ bitstream/handle/11362/5711/S00100849_es.pdf

Avellaneda, P. (2007). Movilidad, pobreza y exclusión social: un estudio de caso en la ciudad de Lima. (Tesis doctoral). Universitat Autònoma de Barcelona, España. http://hdl. handle.net/10803/4969

Bähr, J. \& Borsdorf, A. (2005). La ciudad latinoamericana. La construcción de un modelo. Vigencia y perspectivas. Revista Urbes, 2(2), 207-221. https://www.uibk.ac.at/ geographie/personal/borsdorf/pdfs/urbes-2-2005-207-221.pdf

Borja, J. (2012). Revolución urbana y derechos ciudadanos. Claves para interpretar las contradicciones de la ciudad actual. (Tesis doctoral). Universitat de Barcelona, España. http://hdl.handle.net/2445/42013 
Bourdieu, P. (2000). Efectos de lugar. En P. Bourdieu, La miseria del mundo (pp. 119-124). Fondo de Cultura Económica.

Cabello Quiñones, A. M. (2012). Calidad de vida en la ciudad de Talca, Chile. (Tesis doctoral). Universitat de Barcelona, Espańa. http://tdx.cat/bitstream/handle/10803/96519/ AMCQ_TESIS.pdf?sequence $=1$

Cabrera-Barona, P., Murphy, T., Kienberger, S. \& Blaschke, T. (2015). A multi-criteria spatial deprivation index to support health inequality analyses. International Journal of Health Geographics, 14(11). https://doi.org/10.1186/s12942-015-0004-x

Cabrera-Barona, P., Wei, C. \& Hagenlocher, M. (2016). Multiscale evaluation of an urban deprivation index: Implications for quality of life and healthcare accessibility planning. Applied Geography, 70, 1-10. https://doi.org/10.1016/j.apgeog.2016.02.009

Chaparro Mendivelso, J. (2007). La segregación digital en contexto.Ar@cne.Revista electrónica de recursos en Internet sobre Geografia y Ciencias Sociales, (95). http://www.ub.edu/ geocrit/aracne/aracne-095.htm

Chaparro Mendivelso, J. (2010). Identificación de la segregación digital territorial en Bogotá, Colombia, a partir de la Encuesta de Calidad de Vida, 2007. Cuadernos de Geografía: Revista Colombiana de Geografia, (19), 111-124. https://doi.org/10.15446/rcdg. $\mathrm{n} 19.16856$

Ciccolella, P. (2008). Economía y espacio: ejes de discusión para una geografía y un desarrollo territorial más allá de la globalización. Scripta Nova: Revista Electrónica de Geografía y Ciencias Sociales, 12(270).

Cite-flacso (Centro de Investigaciones de Políticas Públicas y Territorio, Facultad Latinoamericana de Ciencias Sociales, Ecuador). (2015). Propuesta participativa en el ámbito social para la reducción de brechas y el ejercicio de derechos en la zona 9. Secretaría Nacional de Planificación y Desarrollo (sEnPLAdes), Ecuador.

Consejo Nacional de Desarrollo Urbano (CNDU), Chile. (2017). Sistema de Indicadores y Estándares de Calidad de Vida y Desarrollo Urbano. https://cndu.gob.cl/wpcontent/uploads/2018/03/1.-PROPUESTA-SISTEMA-DE-INDICADORES-YEST\%C3\%81NDARES-DE-DESARROLLO-URBANO.pdf

Correa, L. (2010). Algunas reflexiones y posibilidades del Derecho a la Ciudad en Colombia: los retos de la igualdad, la participación y el goce de los derechos. Revista Jurídica de la Universidad de Palermo, 11, 31-74. http://dspace.palermo.edu/dspace/bitstream/1022 6/545/1/11Juridica03.pdf

De Mattos, C. A. (2010). Globalización y metamorfosis metropolitana en América Latina. De la ciudad a lo urbano generalizado. Revista de Geografia Norte Grande, (47), 81-104. http://dx.doi.org/10.4067/S0718-34022010000300005

Delfim, L. \& Martins, I. (2002). A qualidade de vida urbana - o caso da cidade do Porto. FEP Working Papers, 116. Universidade do Porto, Faculdade de Economia do Porto. http:// wps.fep.up.pt/wps/wp116.pdf

Duhau, E. (2013). La división social del espacio metropolitano: Una propuesta de análisis. Nueva Sociedad, (243). https://nuso.org/articulo/la-division-social-del-espacio-metro politano-una-propuesta-de-analisis/

Escobar, L. (2006). Indicadores sintéticos de calidad ambiental: un modelo general para grandes zonas urbanas. Revista EURE - Revista de Estudios Urbano Regionales, 32(96), 73-98. https://doi.org/10.4067/s0250-71612006000200005 
Fainstein, S. (2013). Planificación, justicia y ciudad/Planning, justice and the city. Urban, (06), 7-20. http://polired.upm.es/index.php/urban/article/view/2050

González García, I. (2013). Una aproximación a la definición de la variedad urbana desde la complejidad: aplicación al análisis urbanistico de tres barrios madrileños. (Tesis doctoral). Universidad Politécnica de Madrid, España. http://oa.upm.es/20230/1/ISABEL_ GONZALEZ_P_1-1.pdf

Habitat International Coalition (HIC). (2012). Carta Mundial por el Derecho a la Ciudad. Revista de Paz y Conflictos, (5), 184-196. https://revistaseug.ugr.es/index.php/revpaz/ article/view/473/555

Henríquez, C. (2004). Reflexiones sobre la Ciudad Sostenible, Modelos e Indicadores Urbanos. Historia y Geografía, (18). http://biblioteca-digital.ucsh.cl/greenstone/collect/revista1_ old/index/assoc/HASH01b1/03b76125.dir/Reflexiones\%20sobre\%20la\%20 ciudad\%20sostenible.pdf

Instituto de la Ciudad, Ecuador. (2015). Indice de calidad de vida. http://institutodelaciudad. com.ec/47-indice-calidad-de-vida/82-indice-calidad-de-vida.html

Jiménez Barbosa, W. G. \& González Borrero, J. I. (2014). Calidad de vida urbana: Una propuesta para su evaluación. Revista de Estudios Sociales, (49), 159-175. https://doi. org/10.7440/res49.2014.12

Katzman, R. \& Retamoso, A. (2005). Segregación espacial, empleo y pobreza en Montevideo [LC/G.2266-P]. Revista de la CEPAL, (85), 131-148. https://www.cepal.org/es/ publicaciones/11004-segregacion-espacial-empleo-pobreza-montevideo

Leva, G. (2005). Indicadores de calidad de vida urbana. Teoría y metodología. Universidad Nacional de Quilmes, Buenos Aires. http://hm.unq.edu.ar/archivos_hm/GL_ICVU.pdf

Limonad, E. \& Monte-Mór, R. (2012). Por el derecho a la ciudad, entre lo rural y lo urbano. Scripta Nova: Revista Electrónica de Geografía y Ciencias Sociales, 16. https://revistes. ub.edu/index.php/ScriptaNova/article/view/14813

Linares-García, J., Hernández-Quirama, A. \& Rojas-Betancur, H. M. (2018). Accesibilidad espacial e inclusión social: experiencias de ciudades incluyentes en Europa y Latinoamérica. Civilizar: Ciencias Sociales y Humanas, 18(35), 115-128. https://doi. org/10.22518/usergioa/jour/ccsh/2018.2/a09

Lindenboim, J., Ainstein, L., Liberali, A. M., Acciarri, S., Grané, M., López Imizcoz, C., Pohl Schnake, V. \& Sfich, V. M. (2000). Calidad de vida urbana: Una discusión conceptual. II Jornadas de Geografía de la UNLP, 13 al 15 de septiembre de 2000, La Plata, Argentina. http://www.memoria.fahce.unlp.edu.ar/trab_eventos/ev.13333/ev.13333.pdf

Ludeńa, W. (2006). Ciudad y patrones de asentamiento. Estructura urbana y tipologización para el caso de Lima. Revista EURE - Revista de Estudios Urbano Regionales, 32(95), 37-59. https://doi.org/10.4067/s0250-71612006000100003

Mathivet, C. (2011). El derecho a la ciudad: claves para entender la propuesta de crear "otra ciudad posible”. En A. Sugranyes \& C. Mathivet (Eds.), Ciudades para tod@s. Por el derecho a la ciudad, propuestas y experiencias (2a ed.) (pp. 23-28). Habitat International Coalition (HIC).

Max-Neef, M., Elizalde, A. \& Hopenhayn, M. (1986). Desarrollo a escala humana: una opción para el futuro. Development Dialogue, (número especial). http://habitat.aq.upm.es/ deh/adeh.pdf 
MDMQ (Plan Metropolitano de Ordenamiento Territorial, Quito). (2012). Plan Metropolitano de Ordenamiento Territorial 2012-2022. Secretaría de Desarrollo, Hábitat y Vivienda, Ecuador. https://www.quito.gob.ec/documents/rendicion_ cuentas/AZC/Articulacion_politicas_publicas/PLAN_ORDENAMIENTO_ TERRITORIAL2012.pdf

Molinatti, F. (2013). Segregación residencial socioeconómica en la ciudad de Córdoba (Argentina): Tendencias y patrones espaciales. Revista INVI, 28(79), 61-94. http:// dx.doi.org/10.4067/S0718-83582013000300003

onu-Hábitat. (2010). Estado de las ciudades America Latina y el Caribe. Programa de las Naciones Unidas para los Asentamientos Humanos (onU-Habitat). http://cite. flacsoandes.edu.ec/media/2016/02/onU_Habitat_2010_Estado_de_las_ciudades_ de_America_Latina_y_el_Caribe1.pdf

onu-Hábitat. (2016). La prosperidad en las ciudades de Ecuador. Primer reporte del Índice de Prosperidad Urbana (CPI) para 27 ciudades ecuatorianas. Quito: CAF [Corporación Andina de Fomento] - Banco de Desarrollo de América Latina. https://www.flipsnack. com/Manthra/la-prosperidad-en-las-ciudades-de-ecuador.html

Organisation for Economic Co-operation and Development (oECD). (2008). Handbook on constructing composite indicators: Methodology and user guide. https://www.oecd.org/ sdd/42495745.pdf

Pizarro, R. (2001). La vulnerabilidad social y sus desafíos: una mirada desde América Latina. [LC/L.I490-P]. Serie estudios estadísticos y prospectivos, 6. Comisión Económica para América Latina y el Caribe (CEPAL). http://repositorio.cepal.org/bitstream/ handle/11362/4762/S0102116_es.pdf

Polèse, M. (1998). Economía urbana y regional. Introducción a la relación entre territorio y desarrollo. Libro Universitario Regional (LUR) - Asociación de Editoriales Universitarias de América Latina y el Caribe (eulac) y Agencia de Cooperación Técnica Alemana (GTZ).

Portes, A. \& Roberts, B. R. (2008). Introducción. La ciudad bajo el libre mercado. La urbanización en América Latina durante los años del experimento neoliberal. En A. Portes, B. R. Roberts \& A. Grimson (Eds.), Ciudades latinoamericanas: un análisis comparativo en el umbral del nuevo siglo (pp. 19-74). Universidad Autónoma de Zacatecas, Miguel Ángel Porrúa.

Roberts, B. R. \& Portes, A. (2008). Conclusión. Enfrentando la ciudad del libre mercado. La acción colectiva urbana en América Latina, 1980-2000. En A. Portes, B. R. Roberts \& A. Grimson (Eds.), Ciudades latinoamericanas: un análisis comparativo en el umbral del nuevo siglo (pp. 425-463). Universidad Autónoma de Zacatecas, Miguel Ángel Porrúa.

Rojas Quezada, C. A., Muñiz Olivera, I. \& García-López, M. Á. (2009). Estructura urbana y policentrismo en el área metropolitana de Concepción. Revista EURE - Revista de Estudios Urbano Regionales, 35(105), 47-70. https://doi.org/10.4067/s025071612009000200003

Rueda, S. (Dir.). (2008). Plan especial de indicadores de sostenibilidad ambiental de la actividad urbanistica de Sevilla. Agencia de Ecología Urbana de Barcelona. http://www.upv.es/ contenidos/CAMUNISO/info/U0681581.pdf 
Sabatini, F. (2003). La segregación social del espacio en las ciudades de América Latina. Documentos del Instituto de Estudios Urbanos, Pontificia Universidad Católica de Chile, Serie Azul, 35, 59-70.

Sabatini, F. \& Brain, I. (2008). La segregación, los guetos y la integración social urbana: Mitos y claves. Revista EURE - Revista de Estudios Urbano Regionales, 34(103), 5-26. https:// doi.org/10.4067/s0250-71612008000300001

Segura, R. (2014). El espacio urbano y la (re)producción de desigualdades sociales. Desacoples entre distribución del ingreso y patrones de urbanización en ciudades latinoamericanas. desiguALdades.net Working Paper Series, (65). https://www.desigualdades.net/ Resources/Working_Paper/65-WP-Segura-Online.pdf

Tello Aragay, E. (1999). Principios e indicadores para ciudades más sostenibles. Inguruak: Soziologia Eta Zientzia Politikoaren Euskal Aldizkaria = Revista Vasca de Sociología y Ciencia Politica, (24), 89-98.

Ugalde, V. (2015). Derecho a la ciudad, derechos en la ciudad. Estudios Demográficos y Urbanos, 30(3), 567-595. http://dx.doi.org/10.24201/edu.v30i3.1494

Vicuña, M., Orellana, A., Truffello, R. \& Moreno, D. (2019). Integración urbana y calidad de vida: disyuntivas en contextos metropolitanos. Revista INVI, 34(97), 17-47. https:// doi.org/10.4067/S0718-83582019000300017 